\title{
Observation of coherent many-body Rabi oscillations
}

\author{
Y. O. Dudin, L. Li, F. Bariani and A. Kuzmich`
}

\begin{abstract}
A two-level quantum system coherently driven by a resonant electromagnetic field oscillates sinusoidally between the two levels at frequency $\Omega$ (refs 1,2 ). In dilute gases, the inhomogeneous distributions of both the coupling strength to the field and the interactions between individual atoms reduce the visibility of these so-called Rabi oscillations and may even suppress them completely. However, in the limit where only a single excitation is present, a collective, many-body Rabi oscillation at a frequency $\sqrt{N} \Omega$ arises that involves all $N \gg 1$ atoms, even in inhomogeneous systems ${ }^{3,4}$. When one of the two levels is a strongly interacting Rydberg level, many-body Rabi oscillations emerge as a consequence of a phenomenon known as Rydberg excitation blockade ${ }^{5}$. Here we report initial observations of coherent many-body Rabi oscillations between the ground level and a Rydberg level using several hundred cold rubidium atoms, with a $0.67(10)$ preparation efficiency of the singly-excited many-body state. The strongly pronounced oscillations indicate a nearly complete excitation blockade of the entire mesoscopic ensemble by a single excited atom.
\end{abstract}

A two-level quantum system coherently driven by a quasiresonant electromagnetic field is one of the centrepieces of modern quantum physics. Notably, Rabi oscillations in isolated single atoms or dilute gases form the basis for metrological applications such as atomic clocks and precision measurements of physical constants ${ }^{6}$. A wide array of two-level systems have been realized, with atoms, molecules, nuclei, and Josephson junctions being some of the prominent settings. More than half a century ago Dicke recognized that an atomic ensemble coupled to an electromagnetic field cannot always be treated as a collection of independent atoms ${ }^{3}$. His ground-breaking work gave rise to a rich field of collective atom-field interaction physics ${ }^{7}$.

A key prediction of Dicke's theory is that under certain conditions atom-field coupling is enhanced by a factor $\sim \sqrt{N}$ when compared to one atom. Collectively enhanced atom-field coupling has since been observed in a variety of settings involving either the emission or absorption of radiation. A coherent multi-atom Rabi oscillation at a frequency $\sqrt{N} \Omega$ is a particularly dramatic manifestation of quantum mechanics at work on mesoscopic scales, where an entire ensemble exhibits the dynamical behaviour of a single two-level system. In 2001, Lukin et al. proposed to realize many-body Rabi oscillations in ensembles of atoms driven by a laser tuned to a Rydberg level, and outlined designs for scalable quantum gates for quantum computation and simulation and generation of entangled collective states for metrology beyond the standard quantum limit ${ }^{5}$.

When an atom is promoted into a Rydberg level with principal quantum number $n$, the valence electron is in an orbit that is $\sim n^{2}$ larger than that of the ground-level atom. The atomic dipole moment is correspondingly larger, so that the interaction of two atoms is increased by $\sim n^{4}$ in the dipole-dipole regime and by $\sim n^{11}$ in the van der Waals regime ${ }^{8}$. For $n \simeq 100$ the interactions are sufficiently strong that for two atoms separated by a distance $\sim 10 \mu \mathrm{m}$ the associated energy shift may prevent the second atom from being excited. This excitation blockade mechanism gives rise to an oscillation between the collective ground state $|G\rangle \equiv \prod_{i=1}^{N}|g\rangle_{i}$ and the state $|R\rangle \equiv 1 / \sqrt{N} \sum_{i=1}^{N}|g\rangle_{1} \ldots|r\rangle_{i} \ldots|g\rangle_{N}$ in which one of the $N$ atoms is in the Rydberg level $|r\rangle$, with frequency $\sqrt{N} \Omega$ (refs 4,5 , $7,9,10)$. The average number $\langle N\rangle_{\mathrm{r}}$ of atoms in level $|r\rangle$ is given by:

$$
\langle N\rangle_{\mathrm{r}}=\sin ^{2}(\sqrt{N} \Omega t / 2)
$$

This result holds for an inhomogeneous distribution of atomlight coupling $\Omega_{\mathrm{i}}$ with the modification $\sqrt{N} \Omega \rightarrow \sqrt{\sum_{i=1}^{N} \Omega_{\mathrm{i}}^{2}}$ and $|R\rangle \rightarrow\left(1 / \sqrt{\sum_{i=1}^{N} \Omega_{\mathrm{i}}^{2}}\right) \sum_{i=1}^{N} \Omega_{\mathrm{i}}|g\rangle_{1} \ldots|r\rangle_{i} \ldots|g\rangle_{N}$, see Methods. For two atoms, Rydberg blockade ${ }^{11}$ and the accompanying $\sqrt{2}$ enhancement of the Rabi oscillation frequency ${ }^{12}$ have been observed. Over the past decade significant progress has been made in studying many-atom Rydberg blockade ${ }^{13-21}$, however neither blockade by a single atom nor the many-body Rabi oscillations have been achieved.

Here we report observations of many-body Rabi oscillations for a mesoscopic $(a \simeq 15 \mu \mathrm{m})$ ensemble of rubidium atoms in the regime of Rydberg excitation blockade by just one atom. To achieve this, the interaction strength $\Delta E_{\mathrm{B}} \equiv \Delta_{i j}(a)$ between a pair of atoms at a distance equal to the ensemble size $a$ must be greater than the spectral width $\delta \omega$ of the exciting laser field. The duration of coherent atom-light interaction is limited to $\lesssim 2 \mu \mathrm{s}$ by the finite coherence time of the ground-Rydberg transition caused by atomic motion ${ }^{22}$. Therefore, we choose a high-lying Rydberg level $|r\rangle=\left|102 s_{1 / 2}\right\rangle$ for which $\Delta E_{\mathrm{B}} \gtrsim 5 \mathrm{MHz}$ is sufficiently large. The experimental apparatus has been described elsewhere ${ }^{22}$; the atoms were excited with laser fields of two-photon linewidth $\delta \omega \approx 5 \mathrm{MHz}$ (comparable to $\Delta E_{\mathrm{B}}$ ) while relying on the dephasing of multiply-excited spin waves ${ }^{23-25}$ to generate high-quality single photons. To realize the excitation blockade regime, we lower the laser linewidths to $<100 \mathrm{kHz}$ and employ a longer ( $1 \mu$ s instead of $0.2 \mu \mathrm{s})$ excitation pulse. We reduce the impact of decreasing atomic density due to ballistic expansion of the cloud, and the concomitant smearing of the oscillations, by using a shorter, $50 \mu$ s instead of $200 \mu$ s, sequence of trials for each lattice loading (see Methods).

We prepare a gas of ${ }^{87} \mathrm{Rb}$ atoms of temperature $T \simeq 10 \mu \mathrm{K}$ and of peak density $\rho_{0} \simeq 10^{12} \mathrm{~cm}^{-3}$ in a one-dimensional optical lattice. The lattice is shut off, and the atoms are driven in resonance between the ground $|g\rangle=\left|5 s_{1 / 2}\right\rangle$ and a Rydberg $|r\rangle$ level with the two-photon Rabi frequency $\Omega(\mathbf{r})=\Omega_{1}(\mathbf{r}) \Omega_{2}(\mathbf{r}) /\left(2 \Delta_{1}\right)$ for a duration $\tau=1 \mu \mathrm{s}$, with the corresponding single-atom excitation 


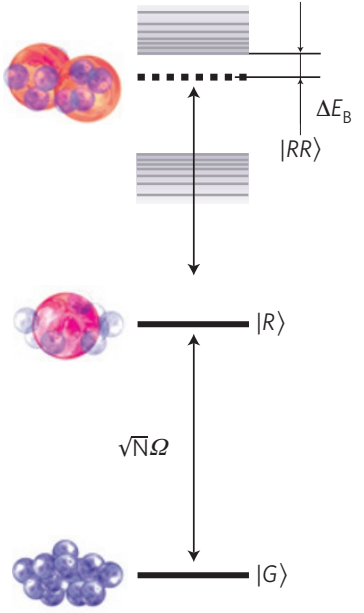

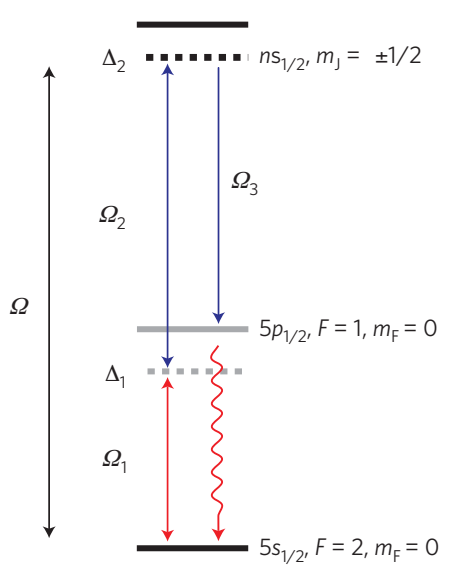

C

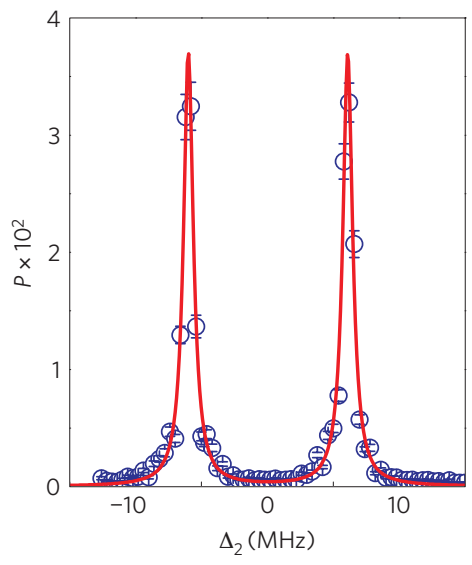

Figure 1 | Rydberg excitation of an atomic ensemble. a, Illustration of the excitation blockade of more than one Rydberg atom in the ensemble. Driving by coherent laser light fields couples the collective ground state $|G\rangle$ to the state with one Rydberg atom $|R\rangle$ with Rabi frequency $\sqrt{N} \Omega$. The doubly excited states $|R R\rangle$ are shifted in energy out of laser resonance by strong atomic interactions. $\mathbf{b}$, Single-atom energy levels for ${ }^{87} \mathrm{Rb}$. Electronic, hyperfine and Zeeman quantum numbers are shown. The detuning from the intermediate $\left|5 p_{1 / 2}\right\rangle$ level is $\Delta_{1}=-40 \mathrm{MHz}$. c, Probability $P$ of photoelectric detection event per trial as a function of two-photon detuning $\Delta_{2}$ for level $\left|102 s_{1 / 2}\right\rangle$. The two $m_{j}= \pm 1 / 2$ Zeeman components are split by the bias magnetic field. The solid curve is a sum of two Lorentzian functions. The $0.9 \mathrm{MHz}$ widths (full-width at half-maximum) of the peaks are determined by the $1 \mu$ s excitation duration.

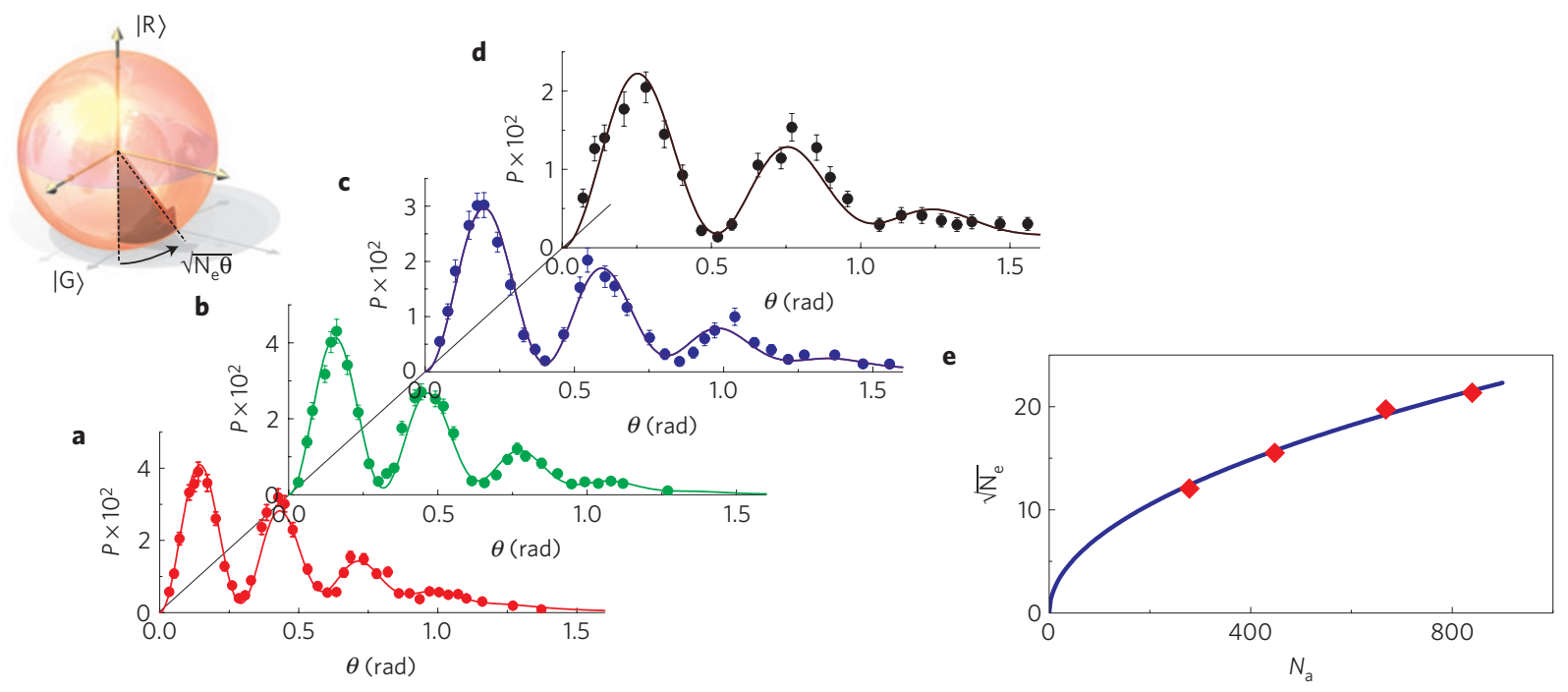

Figure 2 | Coherent many-body Rabi oscillations of a mesoscopic atomic ensemble. a-d, Probability of photoelectric detection $P$ as a function of the

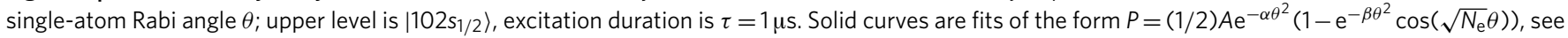
Methods. The fit parameters $\left(A, \alpha, \beta, N_{e}\right)$ are: $(4.3,1.43,1.70,456)(\mathbf{a}),(4.44,1.43,1.77,397)(\mathbf{b}),(3.24,1.14,0.72,243)(\mathbf{c})$ and $(2.56,0.79,0.86,148)$ (d). The error bars represent one standard deviation $(\sqrt{M})$ for $M$ photoelectric counting events. e, $\sqrt{N_{e}}$ as a function of number of atoms $N_{a}$ determined from fluorescence measurements. The data are fit with a function $C \sqrt{N_{a}}$, with the best-fit value $C=0.74$. The inset shows a collective Bloch vector tipped by the angle $\sqrt{N_{\mathrm{e}}} \theta$ on the unit sphere corresponding to the many-atom states $|G\rangle$ and $|R\rangle$.

pulse area $\theta \equiv \Omega(0) \tau$, as shown in Fig. 1a,b. The transverse size (Gaussian waists $w_{x} \approx w_{y} \simeq 6 \mu \mathrm{m}$ ) of the Rydberg excitation region is determined by the overlap of the nearly counter-propagating two-photon excitation laser fields $\Omega_{1}$ at $795 \mathrm{~nm}$ and $\Omega_{2}$ at $474 \mathrm{~nm}$. The longitudinal extent of the ensemble is determined by the sample size of the waist $w_{z} \approx 11 \mu \mathrm{m}$ along $z$.

We measure the population of state $|r\rangle$ by quantum state transfer onto a retrieved light field using a $1 \mu$ s long read-out field $\Omega_{3}$ at $474 \mathrm{~nm}$, in resonance with the $\left|102 s_{1 / 2}\right\rangle \leftrightarrow\left|5 p_{1 / 2}\right\rangle$ transition $^{26,27}$. The retrieved field is coupled into a single-mode fibre followed by a beam splitter and a pair of single-photon detectors $D_{1}$ and $D_{2}$. Figure 2 a shows the sum of the photoelectric detection event probabilities at the two detectors $P \equiv p_{1}+p_{2}$ as a function of the single-atom Rabi angle $\theta$, varied by changing
$\Omega_{1}(0)$ between 0 and $5.5 \mathrm{MHz}$ for a fixed $\Omega_{2}(0)=3.3 \mathrm{MHz}$. The data are fit with the sinusoidal oscillation of equation (1) modified by two Gaussians, as described in the Methods. The choice of the fit function is motivated by a physical picture in which the visibility of the oscillation is smeared by fluctuations of the atom number and the intensities of the laser fields $\Omega_{1}$ and $\Omega_{2}$. The overall decay of the retrieved signal is due to an inhomogeneous distribution of light shifts for atoms in state $|R\rangle$, $\propto N_{\mathrm{e}} \Omega(0)^{2} / \Delta E_{\mathrm{B}}$ which couple the state $|R\rangle$ to other collective singly-excited states $\left|R^{\prime}\right\rangle$, and due to population of doubly-excited states $|R R\rangle$ which are retrieved with substantially suppressed efficiency due to spin-wave dephasing ${ }^{22-24}$. The effective number of atoms $N_{\mathrm{e}}$ is defined as $N_{\mathrm{e}} \equiv \sum_{i=1}^{N} \Omega_{\mathrm{i}}^{2} / \Omega^{2}(0)$. For our experimental geometry $\Omega_{\mathrm{i}}^{2}=\Omega^{2}(0) \exp \left(-2 x^{2} / w_{x}^{2}-2 y^{2} / w_{y}^{2}\right)$, and the atom 


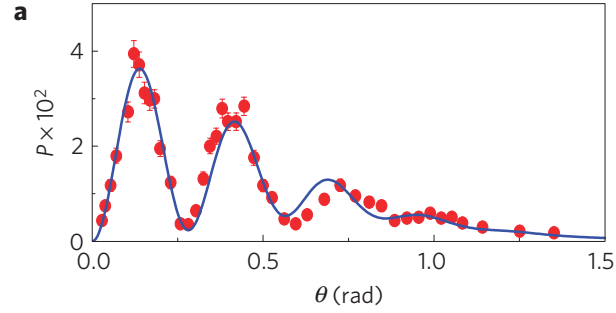

b

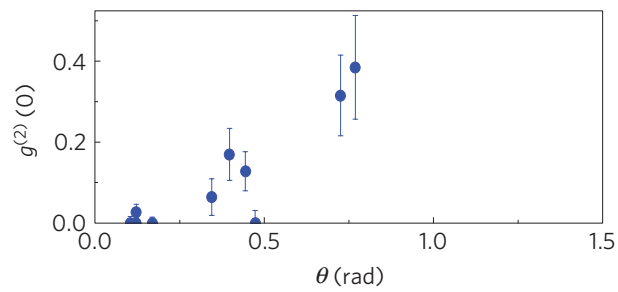

Figure 3 | Quantum statistics of retrieved light as a function of the single-atom Rabi angle $\boldsymbol{\theta}$. Excitation duration is $\tau=1 \mu$ s and upper level is $\left|102 s_{1 / 2}\right\rangle$. a, Probability of photoelectric detection $P$. The solid curve is a fit as in Fig. 2a-d. The fit parameters $\left(A, \alpha, \beta, N_{\mathrm{e}}\right)$ are $(3.80,1.48,1.86,492)$. b. Second-order intensity correlation function at zero delay $g^{(2)}(0)$ as a function of $\theta$. The error bars represent \pm one standard deviation $(\sqrt{M})$ for $M$ photoelectric counting events.

density $\rho=\rho_{0} \exp \left(-2 z^{2} / w_{z}^{2}\right)$. Therefore $N_{\mathrm{e}}=(\pi / 2)^{3 / 2} w_{x} w_{y} w_{z} \rho_{0}$. The efficiency $\eta_{\mathrm{p}}$ with which state $|R\rangle$ is prepared is obtained by normalizing the probability of a photoelectric detection event per trial $P\left(\theta \approx \pi / \sqrt{N_{\mathrm{e}}}\right) \simeq 0.04$ by the retrieval efficiency of state $|R\rangle$ into a single photon $\eta_{\mathrm{r}}=0.23(3)$ and the transmission and detection efficiency $\eta_{\mathrm{td}}=0.26$ (see Methods). At the first oscillation maximum $\left(\theta=\pi / \sqrt{N_{\mathrm{e}}}\right)$ we obtain $\eta_{\mathrm{p}}=0.67(10)$. The uncertainty is largely due to the value of $\eta_{\mathrm{r}}$ measured with the $\left|81 s_{1 / 2}\right\rangle$ Rydberg level. $\eta_{\mathrm{r}}$ maybe be somewhat lower for the $\left|102 s_{1 / 2}\right\rangle$ level owing to longer retrieved fields and correspondingly larger motional dephasing $^{22}$. Our Monte-Carlo simulations of the excitation process that include atomic interactions and motional spin-wave dephasing predict $\eta_{\mathrm{p}} \simeq 0.75$ for $\theta=\pi / \sqrt{N_{\mathrm{e}}}$. We expect measured values of $\eta_{\mathrm{p}}$ to be closer to unity when Rydberg excitation blockade is stronger, which can be achieved by reducing the size of the ensemble or increasing the lifetime of the ground-Rydberg optical coherence.

To explore the collective character of the observed Rabi oscillations, we measure $P$ as a function of $\theta$ while varying the peak density of the sample $\rho_{0}$, as shown in Fig. 2b-d. Figure 2e shows the normalized frequency of the Rabi oscillation $\sqrt{N_{e}}$ extracted from the data in Fig. 2a-d as a function of the number of atoms in the ensemble $N_{\mathrm{a}}$. The latter is calculated using the peak density $\rho_{0}$ measured by the hyperfine state-selective fluorescence imaging of the atomic sample with magneto-optical trap cooling beams used without a repumping field to exclude the contribution of $\left|5 s_{1 / 2}, F=1\right\rangle$ atoms. The absence of further peaks in Fig. 1c supports a near-unity value for the fraction of atoms $f$ in the $m=0$ Zeeman sub-level. Ideally, we expect the effective atom number $N_{\mathrm{e}}$ extracted from the Rabi oscillation period to equal the atom number $N_{\mathrm{a}}$ determined by fluorescence imaging of the sample. The parameter $C$ in the fit in Fig. 2e would equal unity, whereas we extract $C=0.74$. Apart from the factor $\sqrt{f}$, probable causes for $C<1$ are alignment imperfections, uncertainties in the determined waists of the two-photon excitation laser beams, and uncertainties in the fluorescence measurements of $\rho_{0}$.

We further confirm that the dynamics seen in Fig. 2 correspond to the oscillation of equation (1) by measurements of the secondorder intensity correlation function at zero delay $g^{(2)}(0)$ as a function of $\theta$, shown in Fig. 3. Measured values of $g^{(2)}(0)$ well below unity, together with substantial visibility of the oscillations,

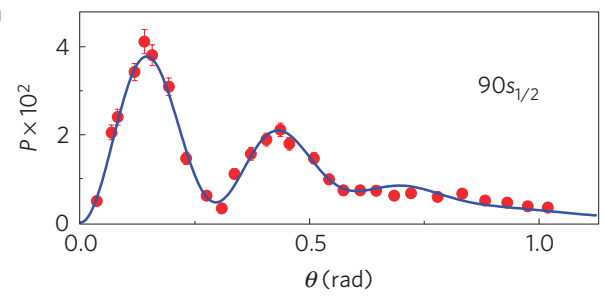

b

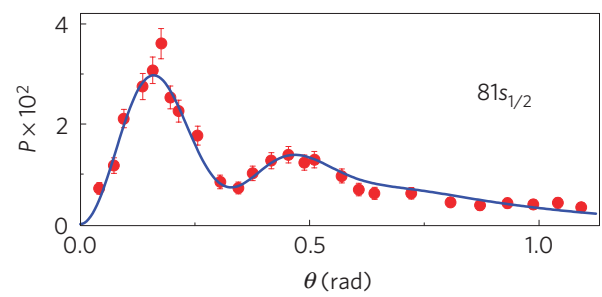

Figure 4 | Probability of photoelectric detection $P$ as a function of the single-atom Rabi angle $\boldsymbol{\theta}$. Excitation duration is $\tau=1 \mu \mathrm{s}$. The solid curves are fits as in Fig. $2 a-d$, where the fit parameters $\left(A, \alpha, \beta, N_{\mathrm{e}}\right)$ are $(4.10,2.00,3.52,441)$ for $n=90$ in $\mathbf{a}$ and $(3.42,1.62,6.70,335)$ for $n=81$ in $\mathbf{b}$, respectively. The error bars represent \pm one standard deviation $(\sqrt{M})$ for $M$ photoelectric counting events.

a

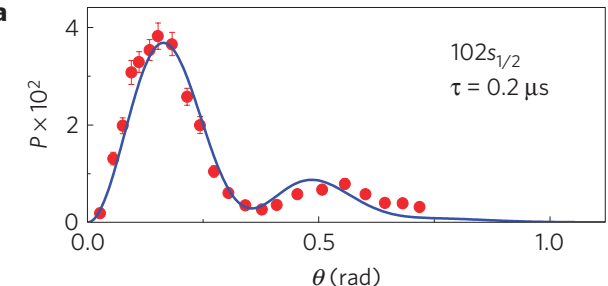

b

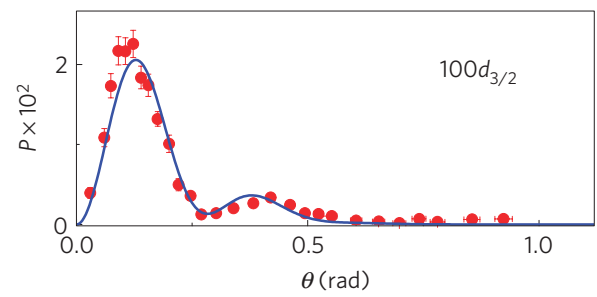

Figure 5 | Probability of photoelectric detection $P$ as a function of the single-atom Rabi angle $\boldsymbol{\theta}$. Level $\left|102 s_{1 / 2}\right\rangle$ is excited for $\tau=0.2 \mu \mathrm{s}$ in $\mathbf{a}$, and level $\left|100 d_{3 / 2}\right\rangle$ is excited for $\tau=1 \mu \mathrm{s}$ in $\mathbf{b}$. The solid curves are fits as in Fig. $2 \mathrm{a}-\mathrm{d}$. The fit parameters $\left(A, \alpha, \beta, N_{\mathrm{e}}\right)$ in a are $(4.56,5.27,3.86,340)$. The excitation spectrum for $\left|100 d_{3 / 2}\right\rangle$ shows a complex structure, probably due to an interplay of an ambient electric field with the bias magnetic field. For the data in $\mathbf{b}$, the laser is tuned to the strongest spectral component, with the scale $\theta^{\prime}$ determined by using the value of $N_{\mathrm{e}}=492$ from the preceding measurements with the $\left|102 s_{1 / 2}\right\rangle$ level, with a fit providing the value of peak single-atom Rabi frequency $\Omega_{0}$ and the fit parameters $(A, \alpha, \beta)$ are $(2.58,10.7,3.49)$. The vertical error bars represent \pm one standard deviation $(\sqrt{M})$ for $M$ photoelectric counting events. The horizontal error bars in $\mathbf{b}$ reflect the uncertainty in determination of the $x$ axis scale $\theta^{\prime}$.

indicate that only one Rydberg excitation is present in the entire ensemble of several hundred atoms. The substantial observed values of $g^{(2)}(0) \approx 0.3$ for $\sqrt{N_{\mathrm{e}}} \theta \geq 5 \pi$ in Fig. $3 \mathrm{~b}$ suggest that the population of doubly-excited states contributes noticeably to the extracted values of $\alpha$. Combining all the data points for $\sqrt{N_{\mathrm{e}}} \theta \approx \pi$ in Fig. 3b we obtain $g^{(2)}(0)=0.006(6)$, which to our knowledge is the lowest value for this quantity for any previously reported light source. It is consistent with a lower bound of 
$g_{b g}^{(2)}(0)=0.012(2)$ due to background counts, of which about half are due to detector dark counts. Our Monte-Carlo simulations suggest that both excitation blockade ${ }^{5}$ and spin-wave dephasing ${ }^{23}$ mechanisms contribute to the suppression of two-photon events. In contrast, in our previous study using shorter and wider linewidth excitation, numerical simulations employing spin-wave dephasing, without excitation blockade, accurately described the observed spatial spin-wave correlation ${ }^{28}$.

The importance of achieving excitation blockade, $\Delta E_{\mathrm{B}} \gg \delta \omega$, to observe many-body Rabi oscillations is checked by reducing $\Delta E_{\mathrm{B}}$ in measurements with $n=90$ and $n=81$, as shown in Fig. 4 . Figure $5 \mathrm{a}$ shows data with increased $\delta \omega$ using a shorter $\tau=0.2 \mu \mathrm{s}$ excitation. The oscillation is less pronounced both for smaller $\Delta E_{\mathrm{B}}$, Fig. 4 , and larger $\delta \omega$, Fig. 5a. Figure 5b shows a similarly suppressed oscillation in measurements with the $\left|100 d_{3 / 2}\right\rangle$ level with a $\tau=1 \mu$ s excitation. This may be attributed to a blockade breakdown due to a strong angular dependence of the atomic interaction strengths for $|n d\rangle$-levels ${ }^{13}$. It should also be noted that for a Gaussian distribution of atom-field couplings single-atom Rabi oscillations are almost completely washed out ${ }^{29}$, which makes the observation of many-atom oscillations under these conditions even more remarkable.

We have demonstrated coherent many-body Rabi oscillations in an ensemble of several hundred cold rubidium atoms. The oscillations provide compelling evidence for the achievement of a collective Rydberg excitation blockade by a single excited atom. Our results pave the way towards quantum computation and simulation using ensembles of atoms ${ }^{5,30}$.

\section{Methods}

Atom preparation and laser excitation. A magneto-optical trap of ${ }^{87} \mathrm{Rb}$ is loaded from background vapour for $70 \mathrm{~ms}$. During the following $25 \mathrm{~ms}$, the detuning of cooling light is increased, the repump intensity is decreased, and the optical lattice is turned on. The lattice is composed of a single $782 \mathrm{~nm}$ retro-reflected linearly polarized Gaussian beam. Untrapped atoms are allowed to fall away from the experimental region during the next $15 \mathrm{~ms}$ period, and a $B_{0}=4.3 \mathrm{G}$ bias magnetic field is turned on. The trapped atoms are optically pumped to the $\left|5 s_{1 / 2}, F=2, m_{F}=0\right\rangle$ state, the optical lattice is switched off by an acousto-optical modulator (AOM), and a $3 \mu$ s long sequence of two-photon Rabi driving and retrieval is repeated for $50 \mu$ s, with a $1 \mu$ s optical pumping period included every five cycles. The overall repetition rate of the experiment is $\approx 8 \mathrm{~Hz}$. For the data in Fig. 2 the peak density $\rho_{0}$ was controlled by varying the time period between lattice loading and the two-photon excitation sequence between 15 and $90 \mathrm{~ms}$. Both $\Omega_{1}$ (at $795 \mathrm{~nm}$ ) and $\Omega_{2}$ (at $474 \mathrm{~nm}$ ) fields are linearly polarized along the same axis. The $795 \mathrm{~nm}$ field is produced by an extended cavity diode laser. Light at $474 \mathrm{~nm}$ is generated by frequency-doubling the output of a tapered amplifier driven by a $950 \mathrm{~nm}$ extended cavity diode laser. Both lasers are frequency-locked to a thermally stabilized ultra-low expansion glass cavity. The transition is located by scanning the laser frequency across a resonance and measuring the photoelectric detection probability for the retrieved field. The 795 and $474 \mathrm{~nm}$ excitation fields are tuned to the two-photon resonance between the ground-level component $\left|5 s_{1 / 2}, F=2, m_{F}=0\right\rangle$ and a Zeeman component of the Rydberg level $\left|n s_{1 / 2}, m_{j}=-1 / 2\right\rangle$. The single-photon Rabi frequency on the blue transition is $\Omega_{2}=-e \mathcal{E}\left\langle 5 p_{1 / 2}, F=1, m_{F}=0|r| n s_{1 / 2}, m_{j}\right\rangle$, where $\mathcal{E}$ is electric field amplitude The radial matrix element is reduced using the Wigner-Eckart theorem. The angular part is calculated following ref. 31 , while the reduced matrix element is approximated by $\langle r\rangle=0.14 \times(50 / n)^{3 / 2} a_{0}$ (ref. 13).

Because $\Omega_{2}$ and $\Omega_{3}$ fields are propagating in the same spatial mode, the retrieved field is phase matched into the mode of the $\Omega_{1}$ field and coupled into a single mode 50/50 fibre beamsplitter followed by a pair of single-photon detectors $D_{1}$ and $D_{2}$. A gating AOM at the fibre beamsplitter input port is employed to avoid damage to the single photon detectors by the $\Omega_{1}$ field.

Photoelectric detection and data analysis. For every experimental trial, photoelectric events on detectors $D_{1}$ and $D_{2}$ are recorded within a time interval determined by the length of the retrieved pulse. Photoelectric detection probabilities for both detectors are calculated as $p_{1,2}=N_{1,2} / N_{0}$, where $N_{1,2}$ are the numbers of recorded events and $N_{0}$ is the number of received triggers.

For the storage and retrieval protocol $p_{1,2} \sim \eta_{\mathrm{s}} \eta_{\mathrm{r}} \eta_{\mathrm{td}} n$, where $n$ is the number of photons in the incident $\Omega_{1}$ field. We can therefore extract the retrieval efficiency of state $|R\rangle$ into a single photon $\eta_{\mathrm{r}}$ from $\eta_{\mathrm{s}} \eta_{\mathrm{r}}$ through the retrieved signal measurements, and the storage efficiency $\eta_{\mathrm{s}}$ through the measurements of the transmitted fraction of $\Omega_{1}$. Using $|r\rangle=\left|81 s_{1 / 2}\right\rangle$ we obtain $\eta_{s}=0.0098(14)$ and $\eta_{\mathrm{s}} \eta_{\mathrm{r}}=0.00222(7)$, resulting in $\eta_{\mathrm{r}}=0.23(3)$. A higher value of the Rabi frequency $\Omega_{2}(0)=5.7 \mathrm{MHz}$ is employed in these measurements. Transmission through the glass vacuum chamber is 0.92 , the gating AOM diffraction efficiency is 0.7 , the fibre coupling efficiency is 0.73 , and the quantum efficiency of the single-photon counters is 0.55 , for a combined light transmission and detection efficiency $\eta_{\text {td }}=0.26$

The photoelectric detection probability for double coincidences is calculated as $N_{12} / N_{0}$, where $N_{12}$ is the total number of simultaneous clicks on both detector for a given experimental trial. The second-order intensity correlation function at zero delay is given by $g^{2}(0)=p_{12} /\left(p_{1} p_{2}\right)$.

Decoherence model. We employ the following Hamiltonian to describe our system: $\hat{H}=\sum_{\mu} \hbar\left(\omega_{g} \hat{\sigma}_{\mu}^{g g}+\omega_{\mathrm{r}} \hat{\sigma}_{\mu}^{r r}\right)+(1 / 2) \sum_{\mu} \hbar\left(\Omega_{\mu} e^{-i \omega_{L} t} \hat{\sigma}_{\mu}^{r g}+\right.$ h.c. $)+$ $\sum_{\mu>v} \hbar \Delta_{\mu \nu} \hat{\sigma}_{\mu}^{r r} \otimes \hat{\sigma}_{v}^{r r}$. The atomic operators for the atom $\mu$ are defined as $\hat{\sigma}_{\mu}^{a b}=|a\rangle_{\mu}\langle b|$, where $a, b \in[g, r]$ with $|g\rangle_{\mu}$ being the atomic ground state and $|r\rangle_{\mu}$ being the addressed Rydberg level. The two-photon excitation is modelled using the effective Rabi frequency $\Omega=\Omega_{1} \Omega_{2} /(2 \Delta)$. The interaction between Rydberg levels is described with a single-channel model. For $\Delta_{\mu \nu} \gg \Omega_{\mu}, \Omega_{\nu} \forall(\mu, \nu)$, the excitation blockade is operational. Adiabatic elimination of double and higher-order excitations from the equations of motion results in an effective Hamiltonian for the singly-excited part of the spectrum: $\hat{H}_{\text {eff }}=\sum_{i} \hbar \Delta_{j}|j\rangle\langle j|+\sum_{i>i} \hbar C_{i j}(|i\rangle\langle j|+| j\rangle\langle i|)+(1 / 2) \sum_{j} \hbar \Omega_{j}(|j\rangle\langle G|+| G\rangle\langle j|)$. Here $\Delta_{j}=-\sum_{i \neq j} \Omega_{i}^{2} /\left(4 \Delta_{i j}\right), C_{i j}=-\Omega_{i} \Omega_{j} /\left(4 \Delta_{i j}\right)$, where $|j\rangle$ is the many-body state with the $j$ th atom in the Rydberg level. The first two terms of the effective Hamiltonian are due to the light shifts induced by the (off-resonant) doubly-excited states onto the single excitations.

When the interaction-induced inhomogeneous light shifts are omitted, the Hamiltonian results in an ideal Rabi oscillation between the ground state $|G\rangle$ and the single spin wave $|R\rangle=\left(1 / \sqrt{\sum_{j} \Omega_{j}^{2}}\right) \sum_{j} \Omega_{j}|j\rangle$. If at time $t=0$ the system is in state $|G\rangle$, the state at future times is given by $|\psi(t)\rangle=\cos (\Omega t / 2)|G\rangle-i \sin (\Omega t / 2)|R\rangle$. When the light shift terms are included, the state $|R\rangle$ is coupled to a broad distribution of singly-excited states and therefore leaks into this quasi-continuum, leading to $P \sim|\langle R \mid \psi(t)\rangle|^{2}$ decaying with a rate $\propto N_{\mathrm{e}} \Omega_{0}^{2} / \Delta E_{\mathrm{B}}$. The doubly-excited states are expected to be populated at a rate $\propto N_{\mathrm{e}} \Omega_{0}^{2}$. Trial-to-trial fluctuations $\Delta \Omega$ and $\Delta N_{\mathrm{e}}$ in $N_{\mathrm{e}}$ and $\Omega_{0}$, respectively, lead to a decay of the oscillation visibility. The probability of photoelectric detection per trial $P$ as a function of $\theta$ in Figs $2-5$ is therefore fit by a function $(1 / 2) A \mathrm{e}^{-\alpha \theta^{2}}\left(1-\mathrm{e}^{-\beta \theta^{2}} \cos \left(\sqrt{N_{\mathrm{e}}} \theta\right)\right)$, where dimensionless fit parameters $\alpha \propto N_{\mathrm{e}}$ and $\beta \propto\left(\Delta N_{\mathrm{e}} / 2 N_{\mathrm{e}}\right)^{2}+\left(\Delta \Omega / \Omega_{0}\right)^{2}$ describe the roles of the light shifts and population of doubly-excited states, and atom number and intensity fluctuations, respectively, while an amplitude $A$ represents the overall measured retrieval and detection efficiency.

Received 28 May 2012; accepted 10 August 2012; published online 9 September 2012

\section{References}

1. Griffiths, D. J. Introduction to Quantum Mechanics (Pearson Prentice Hall, 2005).

2. Rabi, I. I., Millman, S., Kusch, P. \& Zacharias, J. R. The molecular beam resonance method for measuring nuclear magnetic moments. Phys. Rev. 55, 526-535 (1939).

3. Dicke, R. H. Coherence in spontaneous radiation processes. Phys. Rev. 93, 99-110 (1954).

4. Cummings, F. W. \& Dorri, A. Exact solution for spontaneous emission in the presence of N atoms. Phys. Rev. A 28, 2282-2285 (1983).

5. Lukin, M. D. et al. Dipole blockade and quantum information processing in mesoscopic atomic ensembles. Phys. Rev. Lett. 87, 037901 (2001).

6. Ramsey, N. Molecular Beams (Clarendon, 1985).

7. Allen, L. \& Eberly, J. H. Two-level Atoms and Optical Resonance (Dover, 1987)

8. Gallagher, T. F. Rydberg Atoms (Cambridge Univ. Press, 1994),

9. Saffman, M. \& Walker, T. G. Creating single-atom and single-photon sources from entangled atomic ensembles. Phys. Rev. A 66, 065403 (2002).

10. Stanojevic, J. \& Cote, R. Many-body Rabi oscillations of Rydberg excitation in small mesoscopic samples. Phys. Rev. A 80, 033418 (2009).

11. Urban, E. et al. Observation of Rydberg blockade between two atoms. Nature Phys. 5, 110-114 (2009).

12. Gaëtan, A. et al. Observation of collective excitation of two individual atoms in the Rydberg blockade regime. Nature Phys. 5, 115-118 (2009).

13. Saffman, M., Walker, T. G. \& Mølmer, K. Quantum information with Rydberg atoms. Rev. Mod. Phys. 82, 2313-2363 (2010).

14. Singer, K. et al. Suppression of excitation and spectral broadening induced by interactions in a cold gas of Rydberg atoms. Phys. Rev. Lett. 93, 163001 (2004).

15. Tong, D. et al. Local blockade of Rydberg excitation in an ultracold gas. Phys. Rev. Lett. 93, 063001 (2004).

16. Liebisch, T. C., Reinhard, A., Berman, P. R. \& Raithel, G. Atom counting statistics in ensembles of interacting Rydberg atoms. Phys. Rev. Lett. 95, 253002 (2005)

17. Vogt, T. et al. Dipole blockade at Forster resonances in high resolution laser excitation of Rydberg states of cesium atoms. Phys. Rev. Lett. 97, 083003 (2006). 
18. Heidemann, R. et al. Evidence for coherent collective Rydberg excitation in the strong blockade regime. Phys. Rev. Lett. 99, 163601 (2007).

19. Reetz-Lamour, M., Amthor, T., Deiglmayr, J. \& Weidemuller, M. Rabi oscillations and excitation trapping in the coherent excitation of a mesoscopic frozen Rydberg gas. Phys. Rev. Lett. 100, 253001 (2008).

20. Johnson, T. A. et al. Rabi oscillations between ground and Rydberg states with dipole-dipole atomic interactions. Phys. Rev. Lett. 100, 113003 (2008).

21. Viteau, M. et al. Rydberg atoms in one-dimensional optical lattices. Phys. Rev. Lett. 107, 060402 (2011).

22. Dudin, Y. O. \& Kuzmich, A. Strongly interacting Rydberg excitations of a cold atomic gas. Science 336, 887-889 (2012).

23. Bariani, F., Dudin, Y. O., Kennedy, T. A. B. \& Kuzmich, A. Dephasing of multiparticle Rydberg excitations for fast entanglement generation. Phys. Rev. Lett. 108, 030501 (2012).

24. Bariani, F. \& Kennedy, T. A. B. Retrieval of multiple spin waves from a weakly excited, metastable atomic ensemble. Phys. Rev. A 85, 033811 (2012).

25. Stanojevic, J. et al. Generating non-Gaussian states using collisions between Rydberg polaritons. Preprint at http://arxiv.org/abs/1203.6764 (2012)

26. Fleischhauer, M. \& Lukin, M. D. Dark-state polaritons in electromagnetically induced transparency. Phys. Rev. Lett. 84, 5094-5097 (2000).

27. Matsukevich, D. N. \& Kuzmich, A. Quantum state transfer between matter and light. Science 306, 663-666 (2004).

28. Dudin, Y. O., Bariani, F. \& Kuzmich, A. Emergence of spatial spin-wave correlations in a cold atomic gas. Preprint at http://arxiv.org/abs/1205. 4708 (2012).
29. Deiglmayr, J. et al. Coherent excitation of Rydberg atoms in an ultracold gas. Opt. Commun. 264, 293-298 (2006).

30. Weimer, H. et al. A Rydberg quantum simulator. Nature Phys. 6, 382-388 (2010).

31. Walker, T. G. \& Saffman, M. Consequences of Zeeman degeneracy for the van der Waals blockade between Rydberg atoms. Phys. Rev. A 77, 032723 (2008).

\section{Acknowledgements}

We thank A. Radnaev, B. Kennedy and A. Zangwill for discussions. This work was supported by the Atomic Physics Program and the Quantum Memories MURI of the Air Force Office of Scientific Research and the National Science Foundation.

\section{Author contributions}

Y.O.D. and A.K. designed the experiment, Y.O.D. and L.L. carried out the experiment and analysed the data, F.B. carried out the theoretical analysis. All authors contributed to writing the manuscript.

\section{Additional information}

Reprints and permissions information is available online at www.nature.com/reprints. Correspondence and requests for materials should be addressed to A.K.

\section{Competing financial interests}

The authors declare no competing financial interests. 